\title{
A SYMPLECTIC BEAM-BEAM INTERACTION WITH ENERGY CHANGE
}

\author{
KOHJI HIRATA ${ }^{1}$, HERBERT MOSHAMMER ${ }^{2}$ and FRANCESCO RUGGIERO ${ }^{3}$ \\ ${ }^{1}$ KEK, National Laboratory for High Energy Physics, Tsukuba, Ibaraki 305, Japan \\ ${ }^{2}$ SLAC, Stanford Linear Accelerator Center, Stanford University, \\ Stanford, CA 94309, USA \\ ${ }^{3}$ CERN, European Laboratory for Particle Physics, \\ CH1211 Geneva 23, Switzerland
}

(Received 8 September 1992; in final form 8 October 1992)

\begin{abstract}
A mapping, called synchro-beam mapping, is proposed which describes the effects of the beam-beam interaction in presence of synchrotron motion. The mapping is symplectic in six dimensional phase space. In addition to the usual transverse kick, this mapping includes the bunch-length effect on the collision point and the energy variation caused by the electric field of the opposite bunch. The case of weak-strong interaction is investigated by simulation, showing that the beam-beam energy exchange has an influence on the distribution tail.
\end{abstract}

KEY WORDS: Beam-beam interaction, Symplectic map

\section{INTRODUCTION}

The performance of many colliding storage rings is limited by the beam-beam interaction ${ }^{1}$. A particle feels a nonlinear force produced by the encountering bunch at the collision. This beam-beam force acts mainly in the transverse directions so that the longitudinal effects have scarcely been studied ${ }^{2}$, except for the cases of a collision with a crossing angle $^{3}$. Recently, however, high luminosity machines are being considered ${ }^{4,5,6}$ where the beams are focused extensively at the interaction point (IP) so that the beam sizes can vary significantly within the bunch length. Krishnagopal and Siemann ${ }^{7}$ have shown that we should not neglect the bunch length effect in this case. The transverse kick depends on the longitudinal position as well as on the transverse position. If we include this effect, however, from the action-reaction principle, we should expect, at the same time, an energy change which depends on the transverse coordinates. Such an effect is reasonably understood from the fact that the beam-beam force is partly due to the electric field, which can change the energy ${ }^{8,9}$.

The action-reaction principle comes from the symplecticity of the reaction: the electromagnetic influence on a particle is described by a Hamiltonian. The symplecticity is one 
of the most fundamental requirements when studying the beam dynamics. A nonsymplectic approximation can easily lead to unphysical results, in particular in proton rings. Also we will show some noticiable effects in $e^{+} e^{-}$case.

In this paper, we propose a simple, approximate but symplectic mapping for the beambeam interaction which includes the energy change as well as the bunch-length effect. Some preliminary discussion is given in Refs. ${ }^{10,11}$. In the next section, we propose the mapping in a Hamiltonian form, which directly assures its symplecticity. Then in Sect.3, we will study the nature of the mapping by interpreting its consequences. The mapping itself is quite general and can be applied to any distribution function. We show in Sect.4 how it appears when the distribution function is a Gaussian in transverse directions. The mapping is applied to the weak-strong case and some numerical results will be shown in Sect.5. The last section, Sect.6 will be devoted to discussions.

\section{THE SYNCHRO-BEAM MAPPING}

\subsection{Accelerator Coordinates}

The motion of a particle is described by the accelerator coordinates

$$
\boldsymbol{x} \equiv\left(x, p_{x}, y, p_{y}, z, \epsilon\right)
$$

as functions of the distance $s$ from the IP.

In a drift space, i.e. in the absence of any electric or magnetic field, $x$ is related to the local Cartesian coordinates as follows: $x$ and $y$ are two transverse coordinates perpendicular to the direction of $s,\left(p_{x}, p_{y}\right)$ are the corresponding relativistic momenta normalized, i.e.

$$
\left(p_{x}, p_{y}\right)=\frac{m \gamma}{\left|\boldsymbol{p}_{0}\right|}\left(\frac{\mathrm{dx}}{\mathrm{dt}}, \frac{\mathrm{dy}}{\mathrm{dt}}\right),
$$

where $\left|p_{0}\right|$ is the absolute value of the three-momentum $\boldsymbol{p}$ for the reference particle and $\gamma$ is the associated relativistic Lorentz factor. (We do not use the so-called canonical momenta, which include the vector potentials). We use

$$
z=s-c t(s),
$$

where $c$ is the light velocity, $t$ is the difference in the arrival time at $s$ between the considered particle and the reference particle, $(z(s)>0$ means that the particle arrives at $s$ earlier than the reference particle) and

$$
\epsilon=\frac{|\boldsymbol{p}|-\left|\boldsymbol{p}_{0}\right|}{\left|\boldsymbol{p}_{0}\right|}=\frac{E-E_{0}}{E_{0}} .
$$

Here, the ultrarelativistic approximation is used so that $E=c|\boldsymbol{p}|$ holds.

We study the change of $x$, Eq.(1), by the beam-beam interaction. Since both bunches move in opposite directions, we should introduce two kinds of $s, s_{ \pm}$for $e^{ \pm}$bunches. Of course

$$
s_{+}=-s_{-} .
$$

Correspondingly, $z_{+}$and $z_{-}$have opposite directions. 


\subsection{Reduction of the Strong-Strong Problem to the Weak-Strong Problem}

Since we are interested in the strong-strong case, we start with the 6-dimensional distribution functions $\psi_{ \pm}(\boldsymbol{x} ; s)$ for $e^{ \pm}$bunches normalized to unity. We first make longitudinal slices of both bunches. Let us define the longitudinal distribution function $\lambda_{ \pm}(z)$ as

$$
\lambda_{ \pm}(z)=\int \mathrm{dxdydp} \mathrm{dp}_{\mathrm{y}} \mathrm{d} \epsilon \psi_{ \pm}(\boldsymbol{x} ; \mathrm{s})
$$

Each value of $z$ has its transverse plus energy distribution $\tilde{\psi}_{ \pm}\left(x, y, p_{x}, p_{y}, \epsilon ; z_{ \pm}, s\right)$ :

$$
\tilde{\psi}_{ \pm}\left(x, y, p_{x}, p_{y}, \epsilon ; z, s\right)=\frac{\psi_{ \pm}(\boldsymbol{x} ; s)}{\lambda_{ \pm}(z)} .
$$

This defines the distribution function of each longitudinal slice. It seems useful here to note that $\psi(\boldsymbol{x} ; s)$ is not a distribution at a given time (snapshot image) but rather at a given position $s$, (streak image). Thus, the distribution of each slice, $\tilde{\psi}\left(x, y, p_{x}, p_{y}, \epsilon ; z_{ \pm}, s=0\right.$ ), is evaluated when it passes the IP. At the IP $\left(s_{ \pm}=0\right)$, each slice may have $z(0)_{ \pm}$. Slices having the largest $z(0)_{ \pm}$first collide with each other.

Imagine that we have $\psi_{ \pm}\left(s_{ \pm}=0\right)$, which came out of the arc, without being affected by the beam-beam interaction. Actually, some particles should have been affected already. Thus this $\psi$ is an ideal one. The slices having $z_{+}(0)$ and $z_{-}(0)$ collide with each other at

$$
s_{+}=\frac{z_{+}-z_{-}}{2} .
$$

A consequence is that the point where a test particle collides with the slice is not the $\operatorname{IP}(s=0)$. We call this point the collision point $(\mathrm{CP})$. It changes from turn to turn.

To apply the beam-beam interaction, it is convenient to introduce a virtual (or logical) time $\tau$. At $\tau$, slices with $z_{+}$and $z_{-}$such that

$$
z_{+}+z_{-}=-c \tau
$$

collide with each other. This $\tau$ can vary from $-\infty$ to $+\infty$. An event occurring later (with respect to the virtual time) is influenced by the earlier events, but not vice versa. At each $\tau$, we can consider the collision of a pair of slices, ignoring other pairs. Thus the study can be reduced to that of a collision between two slices.

The transverse beam sizes of the slices are kept constant during this collision, because the slice-slice collision takes place in an infinitesimally small time. Thus, when considering the dynamics of an $e^{+}$, for example, the $e^{-}$slice is considered to be unaffected. The beam-beam force then can be treated as a prescribed force. For a single passage problem, thus, the problem is reduced to the so-called weak-strong case. Unless otherwise stated, we will study the effect of one slice (having $z_{*}$ ) on a colliding particle. In this case, the slice is called the strong slice and the particle is called the test particle. Quantities concerning the strong slice will have a subscript $*$ and quantities without $*$ refer to the test particle. 


\subsection{The $4 \times 4$ Treatment}

Before going into detail, it seems convenient to recall the case where the synchrotron motion is negligible. In this case, the system is described by

$$
\boldsymbol{x}_{4} \equiv\left(x, p_{x}, y, p_{y}\right),
$$

and the Hamiltonian for the beam-beam kick can be written as

$$
H_{b b}=N_{*} U\left(x, y ; \mathbf{Q}_{*}(s)\right) \delta(s),
$$

where $N_{*}$ is the number of particles of the strong slice and $\mathbf{Q} \equiv\left(Q_{1}, Q_{2}, \cdots\right)$ represents a set of parameters characterizing the transverse distribution function of the strong slice

$$
\psi(\boldsymbol{x} ; s)=\tilde{\psi}\left(\boldsymbol{x}_{4}, s\right) \delta(s):
$$

since the longitudinal extension is ignored, the whole bunch is represented by a single slice. It is well known that $U$ is essentially the electric scalar potential of the strong slice:

$$
U(x, y ; \mathbf{Q}(s))=-\frac{e e_{*}}{2 \pi \varepsilon_{e} E_{0}} \int \mathrm{d} \boldsymbol{r}_{*} \ln \left(\left|\boldsymbol{r}-\boldsymbol{r}_{*}\right|\right) \tilde{\rho}\left(\boldsymbol{r}_{*} ; \mathbf{Q}(\mathrm{s}), \mathrm{s}\right),
$$

where $\varepsilon_{e}$ is the dielectric constant of the vacuum, $e$ the electric charge of the test particle, $e_{*}$ the same for the particles in the strong slice, $\boldsymbol{r}=(x, y)$, and

$$
\tilde{\rho}(\boldsymbol{r}, s)=\int \mathrm{dp}_{\mathrm{x}} \mathrm{dp}_{\mathrm{y}} \tilde{\psi}\left(\boldsymbol{x}_{4}, \mathrm{~s}\right) .
$$

This Hamiltonian $H_{b b}$ induces a map

$$
\boldsymbol{x}_{4}^{\text {new }}=\exp \left(-N_{*}: U(s=0):\right) \boldsymbol{x}_{4},
$$

at the IP, where $: A:$ denotes the Lie operator ${ }^{12}$ defined by

$$
: A: \boldsymbol{x} \equiv[A, \boldsymbol{x}] .
$$

Here, $[$,$] denotes the Poisson bracket. In this case, the normalized transverse momenta$ $p_{x}$ and $p_{y}$ change as follows:

$$
\begin{gathered}
p_{i}^{\text {new }}=p_{i}-f_{i}\left(x, y ; \mathbf{Q}^{*}(0)\right), \\
f_{i}=N_{*} \frac{\partial U}{\partial x^{i}},
\end{gathered}
$$

( $i$ stands for either $x$ or $y$ ). Transverse positions $x, y$ and longitudinal coordinates $(z, \epsilon)$ do not change.

When $\tilde{\psi}$ is a Gaussian, only

$$
\sigma_{x}^{2}=\left\langle x^{2}\right\rangle, \quad \text { and } \quad \sigma_{y}^{2}=\left\langle y^{2}\right\rangle
$$


are relevant parameters $\mathbf{Q}$. In this case, we have

$$
H_{b b}=N_{*} U\left(x, y ; \sigma_{x}^{*}(s), \sigma_{y}^{*}(s)\right) \delta(s),
$$

and

$$
U\left(x, y ; \sigma_{x}, \sigma_{y}\right)=-\frac{r_{e}}{\gamma_{0}} \int_{0}^{\infty} \frac{\exp \left(-\frac{x^{2}}{2 \sigma_{x}^{2}+u}-\frac{y^{2}}{2 \sigma_{y}^{2}+u}\right)}{\sqrt{2 \sigma_{x}^{2}+u} \sqrt{2 \sigma_{y}^{2}+u}} \mathrm{du},
$$

where $r_{e}$ is the classical electron radius. The explicit form of $f_{i}$, for this case, is known as Bassetti-Erskine formula ${ }^{13}$ :

$$
\begin{gathered}
f_{y}\left(x, y, \sigma_{x}, \sigma_{y}\right)+i f_{x}\left(x, y, \sigma_{x}, \sigma_{y}\right)=\frac{N_{*} r_{e}}{\gamma_{0}} \sqrt{\frac{2 \pi}{\sigma_{x}^{2}-\sigma_{y}^{2}}} \\
\times\left[\mathrm{w}\left(\frac{x+i y}{\sqrt{2\left(\sigma_{x}^{2}-\sigma_{y}^{2}\right)}}\right)-\exp \left(-\frac{x^{2}}{2 \sigma_{x}^{2}}-\frac{y^{2}}{2 \sigma_{y}^{2}}\right) \mathrm{w}\left(\frac{\frac{\sigma_{y}}{\sigma_{x}} x+i \frac{\sigma_{x}}{\sigma_{y}} y}{\sqrt{2\left(\sigma_{x}^{2}-\sigma_{y}^{2}\right)}}\right)\right] .
\end{gathered}
$$

Here $\mathrm{w}$ is the complex error function.

The map induced by $H_{b b}$, Eq.(11), is of course symplectic in $4 \times 4$ (even in $6 \times 6$ ) sense. If synchrotron motion is not negligible, however, we should proceed to $6 \times 6$ treatment.

\subsection{The Synchro-Beam Mapping}

We propose a mapping for a particle-slice interaction, which is generated by

$$
H_{b b}\left(x, p_{x}, y, p_{y}, z, \epsilon ; s\right)=n_{*} U\left(X\left(x, p_{x}, z\right), Y\left(y, p_{y}, z\right) ; \mathbf{Q}_{*}\left(S\left(z, z_{*}\right)\right)\right) \delta(s) .
$$

Here, $n_{*}$ is $N_{*} \lambda_{*}\left(z_{*}\right)$, the number of particles in the strong slice now considered, $U$ is the same as Eq. (13) but the arguments are different:

$$
\begin{aligned}
& X=X\left(x, p_{x}, z\right)=x+p_{x} S\left(z, z_{*}\right), \\
& Y=Y\left(y, p_{y}, z\right)=y+p_{y} S\left(z, z_{*}\right),
\end{aligned}
$$

with $S$ standing for the location of the collision of the test particle $(z)$ with the strong slice $\left(z_{*}\right)$ as seen from the test particle:

$$
S\left(z, z_{*}\right)=\frac{z-z_{*}}{2} .
$$

When $\psi$ is a Gaussian, we have

$$
\mathbf{Q}_{*}(s)=\left(\sigma_{x}^{*}(s), \sigma_{y}^{*}(s)\right) .
$$


Because of the presence of $\delta(s), H_{b b}$ is applied to $x$ at the IP. This gives a map from $\boldsymbol{x}_{I P}$ to $\boldsymbol{x}_{I P}^{\text {new }}$, even if the collision takes place at the CP.

Let us perform the Lie operation.

$$
\boldsymbol{x}_{I P}^{\text {new }}=\exp \left(:-n_{*} U:\right) \boldsymbol{x}_{I P} .
$$

It is convenient to do it in a different canonical basis. Let us introduce a new set of canonical variables $\mathbf{X}=\left(X, P_{X}, Y, P_{Y}, Z, P_{Z}\right)$ by

$$
\begin{array}{ll}
X=x+p_{x} S\left(z, z_{*}\right), & P_{X}=p_{x}, \\
Y=y+p_{y} S\left(z, z_{*}\right), & P_{Y}=p_{y}, \\
Z=z, & P_{Z}=\epsilon-\frac{p_{x}^{2}+p_{y}^{2}}{4}
\end{array}
$$

They are related to the original variables $\boldsymbol{x}$ by

$$
\mathbf{X}=\exp \left(:-D\left[S\left(z, z_{*}\right)\right]:\right) \boldsymbol{x},
$$

where

$$
D[s]=\frac{p_{x}^{2}+p_{y}^{2}}{2} s .
$$

In these new variables, the Lie transformation Eq. (28) can be written as

$$
\mathbf{X}_{I P}^{\text {new }}=\exp \left(:-n_{*} \hat{U}:\right) \mathbf{X}_{I P},
$$

where

$$
\hat{U}=e^{-: D:} U e^{+: D:}=U\left(X, Y, \mathbf{Q}_{*}\left(S\left(Z, z_{*}\right)\right)\right) .
$$

Thus the map is a function only of coordinates.

We find that $(X, Y, Z)$ remain unchanged

$$
(X, Y, Z)_{\text {new }}=(X, Y, Z)
$$

but

$$
\exp \left(:-n_{*} U(X, Y ; Z):\right)\left(\begin{array}{c}
P_{X} \\
P_{Y} \\
P_{Z}
\end{array}\right)=\left(\begin{array}{c}
P_{X}-f_{X}(X, Y ; Z) \\
P_{Y}-f_{Y}(X, Y ; Z) \\
P_{Z}-g(X, Y ; Z)
\end{array}\right)
$$

where

$$
\begin{aligned}
f_{X}(X, Y ; Z) & \equiv n_{*} \frac{\partial}{\partial X} U(X, Y ; Z), \\
f_{Y}(X, Y ; Z) & \equiv n_{*} \frac{\partial}{\partial Y} U(X, Y ; Z), \\
g(X, Y ; Z) & \equiv n_{*} \frac{\partial}{\partial Z} U(X, Y ; Z) .
\end{aligned}
$$

Note that the $Z$ derivative in the last expression is applied through $\mathbf{Q}$ so that it should be written as

$$
g(X, Y ; Z)=g\left(X, Y ; \mathbf{Q}_{*}\left(S\left(Z, z_{*}\right)\right)=n_{*} \sum_{i} \frac{\partial U}{\partial Q_{i}} \frac{d Q_{i}}{d S} \frac{\partial S}{\partial Z}=\frac{n_{*}}{2} \sum_{i} \frac{\partial U}{\partial Q_{i}} \frac{d Q_{i}}{d S} .\right.
$$


Going back to the original variables $\boldsymbol{x}$, we get the explicit form of Eq. (28):

$$
\begin{aligned}
x^{\text {new }}= & x+S\left(z, z_{*}\right) f_{X}(X, Y ; Z) \\
p_{x}^{\text {new }}= & p_{x}-f_{X}(X, Y ; Z) \\
y^{\text {new }}= & y+S\left(z, z_{*}\right) f_{Y}(X, Y ; Z) \\
p_{y}^{\text {new }}= & p_{y}-f_{Y}(X, Y ; Z) \\
z^{\text {new }}= & z \\
\epsilon^{\text {new }}= & \epsilon-\frac{1}{2} f_{X}(X, Y ; Z)\left[p_{x}-\frac{1}{2} f_{X}(X, Y ; Z)\right] \\
& \quad-\frac{1}{2} f_{Y}(X, Y ; Z)\left[p_{y}-\frac{1}{2} f_{Y}(X, Y ; Z)\right] \\
& \quad-g(X, Y ; Z) .
\end{aligned}
$$

The above map, from $x_{I P}$ to $x_{I P}^{n e w}$, is called synchro-beam map, because it includes the coupling between transverse and longitudinal motions due to the beam-beam interaction. It is clear that the synchro-beam mapping is symplectic for any distribution $\psi_{*}$ of the strong bunch, since it can be derived from the general Hamiltonian Eq. (23).

Some points should be noted:

1. We could first derive the equations of motion and integrate them afterwards to obtain $H_{b b}$. Special care should be taken in integrating $d p_{x} / d s$ because it depends also on $p_{x}$. This approach can be found in Ref. ${ }^{11}$.

2. The synchro-beam mapping is applied at the IP $(s=0)$, although the real collision takes place at $s=S\left(z, z_{*}\right)$, called the collision point (CP). This is a favourable point of our mapping: usually, tracking codes provide the development of $\boldsymbol{x}$ for the arc, which is from IP to IP. Thus, it is desirable to apply the beam-beam mapping at the IP.

3. The mapping, Eq. (30), is of course symplectic, but does not correspond to a real evolution of $\boldsymbol{x}$ in a drift space: because, in the drift space, the energy $\epsilon$ could not change. It would be so if $S\left(z, z_{*}\right)$ did not contain any canonical variable. Since, however, it is closely related to the drift map, we call the mapping, Eq. (30), virtual drift mapping.

4. Relatedly, the variable $\boldsymbol{X}$, Eq. (32), can be identified with the coordinate $\boldsymbol{x}$ at the CP. Since, in turn, the CP is a function of $z$, one may be lead to a 'mysterious' expression $z(s(z))$. By relating it to $\boldsymbol{x}_{I P}$ through the virtual drift, we can avoid this confusion.

5. The potential $U$, used in $H_{b b}$, Eq. (23) was related to the electric potential. In the $4 \times 4$ case, we can add any constant to it. In the synchro-beam mapping, it should be treated more stringently. Only $U$, defined by Eq. (13) should be employed. (An exception: addition of a constant to the particle-particle potential is acceptable: it does not lead to any additional $z$-dependent term). For example, an alternative potential ${ }^{14}$ may be used in 4-dimensional treatment but should not be used in the synchro-beam mapping: the z-derivative diverges when $r \rightarrow \infty$.

6. When considering the slice-slice interaction, Eq. (36) insures the energy-momentum conservation of the two-slice system. It is partly due to the ultra-relativistic approximation. There is no long-range longitudinal force. 
7. We have neglected the effect of the solenoid field, which exists almost always because of the needs of high energy experiments. It is, however, a different effect and should be studied separately.

8. By the synchro-beam mapping, the $z$ does not change. This fact justifies our discussion relying on the concept of the slices. Mixture of particles between two slices does not occur during a single passage of the IP. This is due to an approximation of the drift space Hamiltonian which can be justified for usual ring parameters. See discussion given in section 6.1 .

\section{INTERPRETATION OF THE MAPPING}

We have given the map, which is surely symplectic. How reasonable is it? We shall now discuss the physical meaning of our map.

\subsection{Floating Collision Point}

The calculation of the Lie operation, given in the previous section, shows that the whole mapping consists of three successive mappings:

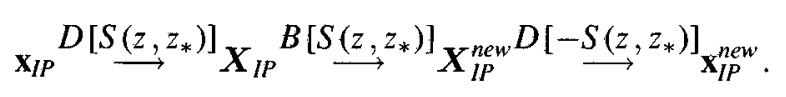

The equation appears to be applied at the IP $(s=0)$, whereas the real action takes place at the CP ( $s=S\left(z, z_{*}\right)$ ). It seems convenient here to give a physical interpretation of the nature of the mapping.

The first step of Eq. (39) is the virtual drift mapping, Eq. (30) from the IP to CP, the second is the beam-beam mapping with respect to the variable $\boldsymbol{X}$, Eq. (32), and the last step is the inverse of the virtual drift mapping. We can say that the whole mapping expresses the collision effect at the CP in terms of the canonical variables defined at the IP.

In this connection, it is worth noting here how to handle the successive collisions with many slices. Imagine that there are $L$ strong slices, each having $n_{i}^{*}$ particles and each having $z_{*}=z_{i}^{*}$. Physically, the test particle first collides with the slice $i=1$, then with 2 ‥L. (We assume $z_{1}^{*}>z_{2}^{*} \cdots z_{L}^{*}$ ). The whole process can be written as

$$
x_{I P}^{\text {new }}=\exp \left(-n_{1}^{*}: U\left(S_{1}\right):\right) \exp \left(-n_{2}^{*}: U\left(S_{2}\right):\right) \cdots \exp \left(-n_{L}^{*}: U\left(S_{L}\right):\right) x_{I P}
$$

where $S_{i}=S\left(z, z_{i}^{*}\right)$. Note the order of the Lie operation: it is the opposite of the physical ordering *.

The effect of the drift from one slice to the next is already included in Eq. (40). With $L$ slices for the strong bunch, we should only apply $\exp \left(-n_{i}^{*}: U\left(S_{i}\right):\right)$ for $L$ times in the order of the virtual time $\tau$. The following formal expression is useful:

$$
x_{I P}^{n e w}=\mathcal{T} \exp \left[-N_{*} \int_{-\infty}^{\infty} d z_{*} \lambda_{*}\left(z_{*}, s=0\right) U\left(S\left(z, z_{*}\right)\right)\right] x_{I P}
$$

* The reason is that the Lie operator operates on the function but not on the numbers. 
where $\mathcal{T}$ denotes the time ordered product with respect to $\tau\left(z, z_{*}\right)$. (From left to right for $\tau$ small to $\tau$ large, with $z$ fixed.). When $\lambda_{*}\left(z_{*}, 0\right)=\delta\left(z_{*}\right)$, Eq. (41) reduces to Eq. (15).

\subsection{The Electromagnetic Field due to a Bunch Slice}

One of the consequences of the fact that the CP is different from the IP is that the electric field has a longitudinal component: even if the IP is a symmetry point, the CP is not.

In this subsection, we will give a physical interpretation of the synchro-beta mapping in terms of the electromagnetic field produced by the strong slice. Thus all quantities should have subscript $*$. For the sake of brevity, however,

in this subsection, the coordinates always follow the sign convention for the strong slices. It is not meant to give the 'exact' derivation of the electromagnetic field: here we should replace $p_{x, y}$ as

$$
\left(p_{x}, p_{y}\right) \longrightarrow\left(x^{\prime}, y^{\prime}\right)=(\mathrm{dx} / \mathrm{ds}, \mathrm{dy} / \mathrm{ds})
$$

In this respect, refer to the discussion given in section 6.1 .

Let

$$
\left(\mathcal{E}_{x}\left(x-x_{1}, y-y_{1}\right), \mathcal{E}_{y}\left(x-x_{1}, y-y_{1}\right)\right) \equiv \frac{e}{2 \pi \varepsilon_{e}} \frac{\boldsymbol{r}-\boldsymbol{r}_{1}}{\left|\boldsymbol{r}-\boldsymbol{r}_{1}\right|^{2}},
$$

be the transverse components of the electric field vector at $\boldsymbol{r}$ produced by an $e^{+}$at $\boldsymbol{r}_{1}$. Here, $\boldsymbol{r}=(x, y)$. Since in the extreme relativistic limit the electric field is perpendicular to the velocity of the source particle, $\mathbf{v}_{1} \propto\left(x_{1}^{\prime}, y_{1}^{\prime}, 1\right)$, we have

$$
\mathcal{E} \cdot \mathbf{v}_{1}=0
$$

and hence

$$
\mathcal{E}_{x} x_{1}^{\prime}+\mathcal{E}_{y} y_{1}^{\prime}+\mathcal{E}_{z}=0
$$

Thus the total integrated electric field produced by the strong slice has components

$$
\begin{aligned}
& \tilde{E}_{x}(\boldsymbol{r})=n \int \mathrm{d} \boldsymbol{r}_{1} \mathcal{E}_{\mathrm{x}}\left(\boldsymbol{r}-\boldsymbol{r}_{1}\right) \tilde{\rho}\left(\boldsymbol{r}_{1}\right), \\
& \tilde{E}_{y}(\boldsymbol{r})=n \int \mathrm{d} \boldsymbol{r}_{1} \mathcal{E}_{\mathrm{y}}\left(\boldsymbol{r}-\boldsymbol{r}_{1}\right) \tilde{\rho}\left(\boldsymbol{r}_{1}\right),
\end{aligned}
$$

and

$$
\tilde{E}_{z}(\boldsymbol{r})=-n \int \mathrm{d} \boldsymbol{r}_{1} \mathrm{~d} \boldsymbol{q}_{1} \mathrm{~d} \varepsilon\left(\mathrm{x}_{1}^{\prime} \mathcal{E}_{\mathrm{x}}\left(\boldsymbol{r}-\boldsymbol{r}_{1}\right)+\mathrm{y}_{1}^{\prime} \mathcal{E}_{\mathrm{y}}\left(\boldsymbol{r}-\boldsymbol{r}_{1}\right)\right) \tilde{\psi}\left(\boldsymbol{r}_{1}, \varepsilon\right),
$$

Here $\boldsymbol{q}_{1}=\left(x_{1}^{\prime}, y_{1}^{\prime}\right)$ and $\tilde{\rho}$ is the distribution function in the coordinate space

$$
\tilde{\rho}(x, y)=\int \mathrm{d} \boldsymbol{q} \mathrm{d} \varepsilon \tilde{\psi}\left(\mathrm{x}, \mathrm{x}^{\prime}, \mathrm{y}, \mathrm{y}^{\prime}, \varepsilon\right), \quad \boldsymbol{q}=\left(\mathrm{x}^{\prime}, \mathrm{y}^{\prime}\right) .
$$

The transverse components $\left(\mathcal{E}_{x}, \mathcal{E}_{y}\right)$ are derivable from

$$
\mathcal{U}\left(\boldsymbol{r}-\boldsymbol{r}_{1}\right)=-\frac{e}{2 \pi \varepsilon_{e}} \ln \left(\left|\boldsymbol{r}-\boldsymbol{r}_{1}\right|\right)
$$


by

$$
\mathcal{E}_{i}(\boldsymbol{r})=-\frac{\partial}{\partial x^{i}} \mathcal{U}\left(\boldsymbol{r}-\boldsymbol{r}_{1}\right)
$$

If we define the total averaged electric potential

$$
\tilde{\varphi}(x, y ; \mathbf{Q}(s))=n \int \mathrm{d} \boldsymbol{r}_{1} \mathcal{U}\left(\boldsymbol{r}-\boldsymbol{r}_{1}\right) \tilde{\rho}\left(\boldsymbol{r}_{1} ; \mathrm{s}\right),
$$

we have

$$
\left(\tilde{E}_{x}(\boldsymbol{r}), \tilde{E}_{y}(\boldsymbol{r})\right)=-\left(\frac{\partial}{\partial x}, \frac{\partial}{\partial y}\right) \tilde{\varphi}(\boldsymbol{r} ; \mathbf{Q}(s)) .
$$

Note that $\tilde{E}$ and $\tilde{\varphi}$ have dimensions of electric field $\times$ length and electric potential $\times$ length, because they are produced by a slice.

Now, we relate $\tilde{\varphi}$ to $\tilde{E}_{z}$, Eq. (48). The distribution function of the strong slice $\tilde{\psi}$ obeys the equation corresponding to the drift space:

$$
\frac{\partial \tilde{\psi}}{\partial s}+x^{\prime} \frac{\partial \tilde{\psi}}{\partial x}+y^{\prime} \frac{\partial \tilde{\psi}}{\partial y}=0
$$

From Eq. (48), we have

$$
\begin{aligned}
\tilde{E}_{z}(\boldsymbol{r}) & =-n \int \mathrm{d} \boldsymbol{r}_{1} \mathrm{~d} \boldsymbol{q}_{1}\left(\mathrm{x}_{1}^{\prime} \mathcal{E}_{\mathrm{x}}\left(\boldsymbol{r}-\boldsymbol{r}_{1}\right)+\mathrm{y}_{1}^{\prime} \mathcal{E}_{\mathrm{y}}\left(\boldsymbol{r}-\boldsymbol{r}_{1}\right)\right) \tilde{\psi}\left(\boldsymbol{r}_{1}, \varepsilon\right) \\
& =+n \int \mathrm{d} \boldsymbol{r}_{1} \mathrm{~d} \boldsymbol{q}_{1}\left(\mathrm{x}_{1}^{\prime} \frac{\partial \mathcal{U}}{\partial x}+\mathrm{y}_{1}^{\prime} \frac{\partial \mathcal{U}}{\partial y}\right) \tilde{\psi}\left(\boldsymbol{r}_{1}, \varepsilon\right) \\
& =-n \int \mathrm{d} \boldsymbol{r}_{1} \mathrm{~d} \boldsymbol{q}_{1}\left(\mathrm{x}_{1}^{\prime} \frac{\partial \mathcal{U}}{\partial x_{1}}+\mathrm{y}_{1}^{\prime} \frac{\partial \mathcal{U}}{\partial y_{1}}\right) \tilde{\psi}\left(\boldsymbol{r}_{1}, \varepsilon\right) \\
& =n \int \mathrm{d} \boldsymbol{r}_{1} \mathrm{~d} \boldsymbol{q}_{1}\left(\mathrm{x}_{1}^{\prime} \frac{\partial \tilde{\psi}}{\partial x_{1}}+\mathrm{y}_{1}^{\prime} \frac{\partial \tilde{\psi}}{\partial y_{1}}\right) \mathcal{U} \\
& =-n \int \mathrm{d} \boldsymbol{r}_{1} \mathrm{~d} \boldsymbol{q}_{1}\left(\partial_{\mathrm{s}} \tilde{\psi}\right) \mathcal{U} \\
& =-\frac{\partial}{\partial s} \tilde{\varphi}
\end{aligned}
$$

In summary, we have

$$
\left(\tilde{E}_{x}, \tilde{E}_{y}, \tilde{E}_{z}\right)=-\left(\frac{\partial}{\partial x}, \frac{\partial}{\partial y}, \frac{\partial}{\partial s}\right) \tilde{\varphi} .
$$

\subsection{Electromagnetic Field Felt by a Test Particle}

We now return to the test particle and recall the convention of $*$. Since $U$ is a scalar function, Eq. (56) holds also true in the coordinate frame of the test particle:

$$
\left(\tilde{E}_{x}, \tilde{E}_{y}, \tilde{E}_{z}\right)=-\left(\frac{\partial}{\partial x}, \frac{\partial}{\partial y}, \frac{\partial}{\partial s_{+}}\right) \tilde{\varphi} .
$$


Since the $U$ in the definition of $H_{b b}$, Eq. (23), is the electric potential, it can be identified with $\tilde{\varphi}$, defined by Eq. (52):

$$
\frac{e \tilde{\varphi}}{E_{0}}=n_{*} U .
$$

from Eq. (13) and Eq. (52). Both are the same thing except for a constant factor.

From Eq. (36), using

$$
\frac{\partial}{\partial Z}=\frac{\partial S}{\partial Z} \frac{\partial}{\partial S}=\frac{1}{2} \frac{\partial}{\partial S}
$$

we can then identify $\left(f_{X}, f_{Y}, g\right)$ as

$$
\left.\begin{array}{rl}
f_{X} & =-\frac{e}{E_{0}} \tilde{E}_{x} \\
f_{Y} & =-\frac{e}{E_{0}} \tilde{E}_{y} \\
g & =-\frac{e}{2 E_{0}} \tilde{E}_{z} .
\end{array}\right\} \text { evaluated at the CP. }
$$

We thus can express the synchro-beam mapping, Eq. (38) as

$$
\begin{aligned}
x^{\text {new }}= & x+\frac{e S\left(z, z_{*}\right)}{E_{0}} \tilde{E}_{x}^{C P}, \\
p_{x}^{\text {new }}= & p_{x}+\frac{e}{E_{0}} \tilde{E}_{x}^{C P}, \\
y^{\text {new }}= & y+\frac{e S\left(z, z_{*}\right)}{E_{0}} \tilde{E}_{y}^{C P}, \\
p_{y}^{\text {new }}= & p_{y}+\frac{e}{E_{0}} \tilde{E}_{y}^{C P}, \\
z^{\text {new }}= & z, \\
\epsilon^{\text {new }}= & \epsilon \frac{e}{2 E_{0}} \tilde{E}_{x}^{C P}\left[p_{x}+\frac{e}{2 E_{0}} \tilde{E}_{x}^{C P}\right] \\
& +\frac{e}{2 E_{0}} \tilde{E}_{y}^{C P}\left[p_{y}-\frac{e}{2 E_{0}} \tilde{E}_{y}^{C P}\right] \\
& +\frac{e}{2 E_{0}} \tilde{E}_{z}^{C P} .
\end{aligned}
$$

The change of the transverse momenta is due to the transverse electric field. Actually, the magnetic field gives the same effect. Thus the transverse electric effect should be multiplied by 2 but it should be divided by 2 again due to the fact that the test particle crosses the strong slice in half the time it would take if the slice did not move and sit at the CP. The latter effect will be refereed to as the 'time-of-flight' effect. The change of transverse coordinates is due to the floating CP. The change of $\epsilon$ is more understandable in the form

$$
\begin{aligned}
\delta \epsilon & =\frac{e}{E_{0}}\left(\frac{1}{2} \tilde{E}_{x}^{C P} \frac{x_{\text {old }}^{\prime}+x_{\text {new }}^{\prime}}{2}+\frac{1}{2} \tilde{E}_{y}^{C P} \frac{y_{\text {old }}^{\prime}+y_{\text {new }}^{\prime}}{2}+\frac{1}{2} \tilde{E}_{z}^{C P}\right) \\
& \simeq \frac{e}{E_{0}}\left(\frac{1}{2} \tilde{E}_{x}^{C P} x^{\prime}+\frac{1}{2} \tilde{E}_{y}^{C P} y^{\prime}+\frac{1}{2} \tilde{E}_{z}^{C P}\right)
\end{aligned}
$$

Because of the electric field, the test particle can change its energy as

$$
\frac{d \epsilon}{d t}=\frac{e}{E_{0}} \mathbf{E} \cdot \mathbf{v}=\frac{c e}{E_{0}}\left(E_{x} x^{\prime}+E_{y} y^{\prime}+E_{z}\right) .
$$

The magnetic field does not contribute to this effect. Because of the time-of-flight effect, we have an extra factor (1/2) in Eq. (62). To the best of our knowledge, this effect was 
first realized by Bassetti ${ }^{8}$, although the possible presence of $E_{z}$ was not considered at that time. Augustin ${ }^{2}$ pointed out the possibility of energy change due to the beam-beam force. His argument, however, was not clear enough. He asserted that, in the rest frame of the strong slice, the change of momentum of the test particle is always perpendicular to its momentum. We should say, instead, the change is always parallel to the electric field.

We have presented the synchro-beam mapping, which is symplectic, and have shown that it allows a reasonable physical interpretation within an approximation that $\left(p_{x}, p_{y}\right) \simeq$ $\left(x^{\prime}, y^{\prime}\right)$. (See discussion given in section 6.1.)

\section{GAUSSIAN DISTRIBUTION}

The discussion thus far is quite general: it applies to an arbitrary distributions of the strong slice. It is useful, however, to see how the synchro-beam mapping looks like for simple but realistic distributions. Here we consider the round Gaussian and bi-Gaussian distributions. In the following calculation, there is no need to specify the longitudinal distribution $\lambda_{*}\left(z_{*}\right)$.' This becomes necessary only when we calculate the successive application of the synchro-beam mapping as in Eq. (41).

\subsection{Round Gaussian Distribution}

The round-Gaussian distribution provides us the simplest non-trivial example, although this might me still too simple as a realistic model. We will use it for the illustration of the mapping and later for the weak-strong simulation. The distribution function is parametrized as

$$
\tilde{\psi}\left(r, p_{r}, s\right)=\frac{1}{2 \pi \sqrt{\operatorname{det} \Sigma(s)}} \exp \left[-\frac{1}{2}\left(r, p_{r}\right) \Sigma(s)^{-1}\left(r, p_{r}\right)^{t}\right]
$$

where $\Sigma$ is a $2 \times 2$ matrix

$$
\Sigma=\left(\begin{array}{cc}
\left\langle r^{2}\right\rangle & \left\langle r p_{r}\right\rangle \\
\left\langle r p_{r}\right\rangle & \left\langle p_{r}^{2}\right\rangle
\end{array}\right)
$$

In this model, we assume a perfect symmetry with respect to the rotation in the $x-y$ plane and consider only one radial direction $r=\sqrt{x^{2}+y^{2}}$. We also omitted the $\varepsilon$ dependence of $\tilde{\psi}$ because it is irrelevant in calculating the beam-beam force.

Since, in a drift space,

$$
r(s)=r(0)+p_{r}(0) s, \quad p_{r}(s)=p_{r}(0),
$$

we have

$$
\begin{aligned}
& \Sigma_{11}(s)=\Sigma_{11}(0)+2 \Sigma_{12}(0) s+\Sigma_{22}(0) s^{2}, \\
& \Sigma_{12}(s)=\Sigma_{12}(0)+\Sigma_{22}(0) s, \\
& \Sigma_{22}(s)=\Sigma_{22}(0) .
\end{aligned}
$$

Thus the envelope of $\tilde{\psi}(s)$ is uniquely determined by that of $\tilde{\psi}(0)$. These formulae can be used to evaluate the beam-beam force at the CP. 
The potential $U$ is given by

$$
U\left(r ; \Sigma_{11}\left(S\left(z, z_{*}\right)\right)=-\frac{r_{e}}{\gamma_{0}} \int_{0}^{\infty} \mathrm{dt} \frac{\exp \left(\frac{-r^{2}}{2 \Sigma_{11}\left(S\left(z, z_{*}\right)\right)+t}\right)}{2_{11}\left(\mathrm{~S}\left(\mathrm{z}, \mathrm{z}_{*}\right)\right)+\mathrm{t}} .\right.
$$

The radial kick at the $\mathrm{CP}$ is

$$
\left(f_{X}, f_{Y}\right)=\frac{(x, y)}{r} f_{r}, \quad f_{r}=n_{*} \frac{\partial U}{\partial r} .
$$

It can be written as

$$
f_{r}=-\frac{2 n_{*} r_{e}}{\gamma_{0} r}\left[\exp \left(\frac{-r^{2}}{2 \Sigma_{11}\left(S\left(z, z_{*}\right)\right)}\right)-1\right] .
$$

On the other hand, according to $\operatorname{Eqs}(36)$ and (37), we have

$$
g=n_{*} \frac{1}{2} \frac{\mathrm{d}_{11}}{\mathrm{ds}} \frac{\partial}{\partial \Sigma_{11}} U=n_{*} \frac{1}{\Sigma_{11}} \frac{\mathrm{d}_{11}}{\mathrm{ds}} \frac{N r_{e}}{2 \gamma_{0}}\left[\exp \left(\frac{-r^{2}}{2 \Sigma_{11}}\right)\right] .
$$

In Eqs. (70) and (71), we use Eq. (67) to evaluate $\Sigma_{11}\left(S\left(z, z_{*}\right)\right)$.

We substitute $\left(f_{X}, f_{Y}, g\right)$ thus obtained into Eq. (38) to obtain the synchro-beam mapping in the present case:

$$
\begin{aligned}
r^{\text {new }} & =r+S\left(z, z_{*}\right) f_{r} \\
p_{r}^{\text {new }} & =p_{r}-f_{r} \\
z^{\text {new }} & =z \\
\epsilon^{\text {new }} & =\epsilon-\frac{1}{2} f_{r}\left(p_{r}-\frac{1}{2} f_{r}\right)-g
\end{aligned}
$$

where

$$
\begin{gathered}
f_{r}=\frac{2 n_{*} r_{e}}{\gamma_{0}} \frac{1}{r+S\left(z, z_{*}\right) p_{r}}\left[1-\exp \left(-\frac{\left(r+S\left(z, z_{*}\right) p_{r}\right)^{2}}{2 \Sigma_{11}\left(S\left(z, z_{*}\right)\right)}\right)\right], \\
g=\left[\frac{1}{\Sigma_{11}} \frac{\mathrm{d}_{11}}{\mathrm{ds}}\right]_{S=S\left(z, z_{*}\right)} \times \frac{n_{*} r_{e}}{2 \gamma_{0}} \exp \left(-\frac{\left(r+S\left(z, z_{*}\right) p_{r}\right)^{2}}{2 \Sigma_{11}\left(S\left(z, z_{*}\right)\right)}\right) .
\end{gathered}
$$

When studying the weak-strong effect, where one of the beams is considered to consist of only one particle so that the other beam can be assumed to be unaffected, we can use

$$
\Sigma(s)=\varepsilon_{0}\left(\begin{array}{cc}
\beta(s) & -\alpha(s) \\
-\alpha(s) & \gamma(s)
\end{array}\right) .
$$

Here, $\varepsilon_{0}$ is the nominal emittance and $(\alpha, \beta, \gamma)$ are the Twiss functions. On the contrary, in the more realistic strong-strong case, the beam envelope $\Sigma$ is perturbed by the beambeam force, partly through the dynamic beta ${ }^{15}$ and the dynamic emittance ${ }^{16}$ effects. As long as the IP and its surrounding can be assumed to be a free drift space, Eq. (67) holds in any case.

We have derived the synchro-beam mapping using direct differentiation of $U$. Note that there is an alternative way to obtain $\left(f_{X}, f_{Y}, g\right)$ based on a calculation of the electric field ${ }^{10}$. Although, of course, the resulting mapping is the same, the calculation shows us that our interpretation of the mapping was right. 


\subsection{The Bi-Gaussian Distribution}

In the bi-Gaussian case, the direct evaluation of the longitudinal field seems quite complicated. We derive the term $g$ by a direct differentiation of $U$, according to Eq. (37). The transverse forces $f_{X}$ and $f_{Y}$ are already known: they are given by Eq. (22) with $\sigma$ 's evaluated at the $\mathrm{CP}$.

Without loss of generality we can assume that $\sigma_{x}>\sigma_{y}$. According to Eq. (37), the total effect of the longitudinal electric field is described by

$$
g\left(x, y, \Sigma_{x}, \Sigma_{y}\right)=\frac{n_{*}}{2}\left(\frac{\mathrm{d}_{\mathrm{x}}}{\mathrm{ds}} \frac{\partial U}{\partial \Sigma_{x}}+\frac{\mathrm{d}_{\mathrm{y}}}{\mathrm{ds}} \frac{\partial U}{\partial \Sigma_{y}}\right) .
$$

We thus have to evaluate

$$
\frac{\partial}{\partial \Sigma_{x}} U\left(x, y, \Sigma_{x}, \Sigma_{y}\right), \text { and } \frac{\partial}{\partial \Sigma_{y}} U\left(x, y, \Sigma_{x}, \Sigma_{y}\right) .
$$

Here, we have introduced an abbreviation $\Sigma_{11}^{x, y}=\Sigma_{x, y}$. Using the following substitutions

$$
a=\frac{x}{\sqrt{2\left(\sigma_{x}^{2}-\sigma_{y}^{2}\right)}}, \quad b=\frac{y}{\sqrt{2\left(\sigma_{x}^{2}-\sigma_{y}^{2}\right)}}, \quad t^{2}=\frac{2 \Sigma_{y}+u}{2 \Sigma_{x}+u}, \quad r=\frac{\sigma_{x}}{\sigma_{y}},
$$

the beam-beam potential Eq. (21) becomes

$$
U(a, b, r)=2 \frac{r_{e}}{\gamma_{0}} \int_{r}^{1} \frac{e^{a^{2}\left(t^{2}-1\right)+b^{2}\left(1-1 / t^{2}\right)}}{\left(t^{2}-1\right)} \mathrm{dt}
$$

and the derivatives of the potential can be written ${ }^{\dagger}$ as

$$
\begin{aligned}
& \frac{\partial}{\partial \Sigma_{x}} U\left(x, y, \Sigma_{x}, \Sigma_{y}\right)=\frac{2 r_{e}}{\gamma_{0}\left(\Sigma_{x}-\Sigma_{y}\right)} I_{x}(a, b, r), \\
& \frac{\partial}{\partial \Sigma_{y}} U\left(x, y, \Sigma_{x}, \Sigma_{y}\right)=\frac{2 r_{e}}{\gamma_{0}\left(\Sigma_{x}-\Sigma_{y}\right)} I_{y}(a, b, r),
\end{aligned}
$$

where the integrals $I_{x}$ and $I_{y}$ are given by

$$
\begin{gathered}
I_{x}(a, b, r)=\int_{r}^{1} e^{a^{2}\left(t^{2}-1\right)+b^{2}\left(1-1 / t^{2}\right)}\left[a^{2}\left(t^{2}-1\right)+1 / 2\right] \mathrm{dt}, \\
I_{y}(a, b, r)=\int_{r}^{1} e^{a^{2}\left(t^{2}-1\right)+b^{2}\left(1-1 / t^{2}\right)}\left[b^{2}\left(t^{2}-1\right) / t^{2}+1 / 2\right] \frac{1}{t^{2}} \mathrm{dt} .
\end{gathered}
$$

† These derivatives should be computed using the original form of the potential $U\left(x, y, \Sigma_{x}, \Sigma_{y}\right)$, Eq. (21), and then performing the change of variables. Otherwise, in differentiating Eq. (79) directly, one should be careful to take the limit for $t \rightarrow 1$ in the upper extreme of the integral (corresponding to $u \rightarrow \infty$ ): this can be done only after the derivatives have been computed. 
Integrals $I_{x}$ and $I_{y}$ are proportional to the second order derivatives of $U(a, b, r)$ with respect to $a$ or to $b$. Indeed from Eq. (79), we have

$$
\frac{\partial U}{\partial \Sigma_{x}}=\frac{1}{4\left(\Sigma_{x}-\Sigma_{y}\right)} \frac{\partial^{2} U}{\partial a^{2}}, \quad \quad \frac{\partial U}{\partial \Sigma_{y}}=\frac{1}{4\left(\Sigma_{x}-\Sigma_{y}\right)} \frac{\partial^{2} U}{\partial b^{2}} .
$$

Recalling that

$$
f_{y}+i f_{x}=n_{*}\left(\frac{\partial}{\partial y}+i \frac{\partial}{\partial x}\right) U=\frac{n_{*}}{\sqrt{2\left(\Sigma_{x}-\Sigma_{y}\right)}}\left(\frac{\partial}{\partial b}+i \frac{\partial}{\partial a}\right) U,
$$

and using Eq. (22), we finally obtain

$$
\begin{gathered}
n_{*} \frac{\partial U}{\partial \Sigma_{x}}=-\frac{1}{2\left(\Sigma_{x}-\Sigma_{y}\right)}\left\{x f_{x}+y f_{y}+\frac{2 n_{*} r_{e}}{\gamma_{0}}\left[\frac{\sigma_{y}}{\sigma_{x}} e^{-\left(\frac{x^{2}}{2 \Sigma_{x}}+\frac{y^{2}}{2 \Sigma_{y}}\right)}-1\right]\right\} \\
n_{*} \frac{\partial U}{\partial \Sigma_{y}}=\frac{1}{2\left(\Sigma_{x}-\Sigma_{y}\right)}\left\{x f_{x}+y f_{y}+\frac{2 n_{*} r_{e}}{\gamma_{0}}\left[\frac{\sigma_{x}}{\sigma_{y}} e^{-\left(\frac{x^{2}}{2 \Sigma_{x}}+\frac{y^{2}}{2 \Sigma_{y}}\right)}-1\right]\right\} .
\end{gathered}
$$

Note that these two expressions tend to 0 when $(x, y) \rightarrow \infty$.

Replacing $\left(x, y, \Sigma_{x}, \Sigma_{y}\right)$ in Eq. (76) and above expressions by $\left(X, Y, \Sigma_{x}(S), \Sigma_{y}(S)\right)$, we obtain the necessary expression.

\section{WEAK-STRONG SIMULATION FOR ROUND GAUSSIAN BEAM}

In this section, we will investigate the numerical consequences of the synchro-beam mapping. Although it is not meant to give fairly realistic and practical predictions, it would be interesting to see some numerical results. Here we restrict ourselves to the simplest case: we study the round Gaussian beam and assume the weak-strong situation. The code includes betatron and synchrotron oscillations with damping and noise, associated with synchrotron radiation in the arcs of an $e^{+} e^{-}$storage ring. It also contains the bunch length effect of the strong bunch.

\subsection{The Program}

The program tracks each particle as follows:

5.1.1 Synchro-Beam Interaction For the sake of brevity, we rescale the canonical variables as follows:

$$
Q=\frac{r}{\sigma_{r}^{0}}, \quad P=\beta_{I P} \frac{p_{r}}{\sigma_{r}^{0}}, \quad Z=\frac{z}{\sigma_{z}^{0}}, \quad \mathcal{E}=\frac{\epsilon}{\sigma_{\epsilon}^{0}}
$$


where $\sigma_{r}^{0}, \sigma_{z}^{0}$ and $\sigma_{\epsilon}^{0}$ are the nominal values at IP of the transverse beam size, bunch length and relative energy spread, respectively. Note that the transformation to these variables is not symplectic. In terms of these variables, the synchro-beam mapping for a collision between a particle in the weak beam and a strong slice having $z_{*}$ is expressed as

$$
\begin{aligned}
& Q \longrightarrow Q-R_{1}\left(Z-Z_{*}\right) \delta P \\
& P \longrightarrow P+\delta P \\
& Z \longrightarrow Z \\
& \mathcal{E} \longrightarrow \mathcal{E}+R_{2} \delta P(P+\delta P / 2)-G
\end{aligned}
$$

where

$$
\begin{gathered}
\delta P \equiv 8 \pi \eta \frac{n_{*}}{N_{*}} \frac{1}{Q+R_{1}\left(Z-Z_{*}\right) P}\left[\exp \left(-A_{c o l}^{2}\right)-1\right], \\
G \equiv 8 \pi \eta \frac{n_{*}}{N_{*}} R_{1} R_{2} \frac{Z-Z_{*}}{1+R_{1}^{2}\left(Z-Z_{*}\right)^{2}} \exp \left(-A_{c o l}^{2}\right), \\
A_{c o l}=\frac{\left(Q+R_{1}\left(Z-Z_{*}\right) P\right)}{\sqrt{2\left(1+R_{1}^{2}\left(Z-Z_{*}\right)^{2}\right)}}, \\
R_{1}=\frac{\sigma_{z}^{0}}{2 \beta_{I P}}, \quad R_{2}=\frac{\varepsilon_{0}}{2 \sigma_{\epsilon}^{0} \beta_{I P}}, \quad Z_{*}=\frac{z_{*}}{\sigma_{z}^{0}} .
\end{gathered}
$$

Here, $\eta$ is the nominal beam-beam parameter:

$$
\eta=\frac{N_{*} r_{e}}{4 \pi \gamma \varepsilon_{0}}
$$

where $\varepsilon_{0}$ is the transverse nominal emittance of the strong beam. The denominator of $A_{c o l}$ represents the variation of the beam size (of the strong slice) due to the floating effect of the CP. See Eq. (67).

5.1.2 Bunch Length Effect The necessity of introducing multiple slices was stressed by Krishnagopal and Siemann?

In the multiparticle tracking, the longitudinal distribution function in Eq. (6) can be replaced by

$$
\lambda_{*}(z)=\sum_{i+1}^{L} w_{i} \delta\left(z-z_{i}^{*}\right), \quad \sum w_{i}=1 .
$$

We consider $L$ longitudinal slices. Let us make these slices in such a way that each slice has the same number of particles $\left(w_{i}=\right.$ const $)$ and let us represent each slice by its longitudinal barycentre. The i-th slice then sits at

$$
z_{i}^{*}=\frac{L}{\sqrt{2 \pi}}\left(\exp \left(-\left(z_{i, i+1}^{*}\right)^{2} / 2\right)-\exp \left(-\left(z_{i-1, i}^{*}\right)^{2} / 2\right)\right)
$$

where $z_{i, i+1}^{*}$ is the border between $\mathrm{i}$-th and (i+1)-th slices. For example, when $L=5$, we have

$$
z_{i}^{*}(\text { barycentre }):-1.3998, \quad-0.5319, \quad 0, \quad 0.5319, \quad 1.3998,
$$




$$
z_{i, i+1}^{*} \text { (border) : } \quad-0.84159, \quad-0.2533,0.2533, \quad 0.84159 .
$$

Each barycentre represents $1 / 5$ of the whole particles.

5.1.3 Oscillation The transformations from IP to IP are just rotations with damping and quantum diffusion.

Thus we first apply the mapping

$$
\begin{aligned}
& \left(\begin{array}{c}
Q \\
P
\end{array}\right) \longrightarrow U\left(2 \pi \nu_{x}\right)\left(\begin{array}{c}
Q \\
P
\end{array}\right), \\
& \left(\begin{array}{c}
Z \\
\mathcal{E}
\end{array}\right) \longrightarrow U\left(-2 \pi \nu_{s}\right)\left(\begin{array}{c}
Z \\
\mathcal{E}
\end{array}\right),
\end{aligned}
$$

where

$$
U(\alpha)=\left(\begin{array}{cc}
\cos \alpha & \sin \alpha \\
-\sin \alpha & \cos \alpha
\end{array}\right)
$$

5.1.4 Radiation For transverse diffusion, the symmetric prescription ${ }^{16}$ is used, which is suitable for the case where the integer part of the betatron tune is much larger than unity. The longitudinal diffusion should be applied only to $\mathcal{E}$, because $\nu_{s}$ is usually much smaller than unity. Thus,

$$
\begin{gathered}
Q \longrightarrow \lambda_{x} Q+\sqrt{1-\lambda_{x}^{2}} \hat{r}_{1}, \\
P \longrightarrow \lambda_{x} P+\sqrt{1-\lambda_{x}^{2}} \hat{r}_{2}, \\
\mathcal{E} \longrightarrow \lambda_{s}^{2} \mathcal{E}+\sqrt{1-\lambda_{s}^{4}} \hat{r}_{3},
\end{gathered}
$$

where the $\hat{r}^{\prime} s$ are independent, random Gaussian variables with unit standard deviation. Here the $\lambda$ 's are damping factors defined by

$$
\lambda_{x}=\exp \left(-1 / T_{x}\right), \quad \text { and } \quad \lambda_{s}=\exp \left(-1 / T_{\epsilon}\right),
$$

where the T's denote damping times normalized by the revolution time, or equivalently the number of beam-beam collisions per betatron and synchrotron damping times, respectively.

5.1.5 Independent Parameters In the synchro-beam mapping, we introduced many variables. The essential parameters, however, are not so many. In the round-Gaussian case, the only dimensionless free parameters are the following:

- Nominal beam-beam parameter $\eta$, which is proportional to the total number of particles in the strong bunch.

- Transverse and synchrotron tunes $\nu_{x}$ and $\nu_{s}$,

- Transverse and syńchrotron damping times $T_{x}$ and $T_{\epsilon}$,

- Ratios $R_{1}$ and $R_{2}$. 


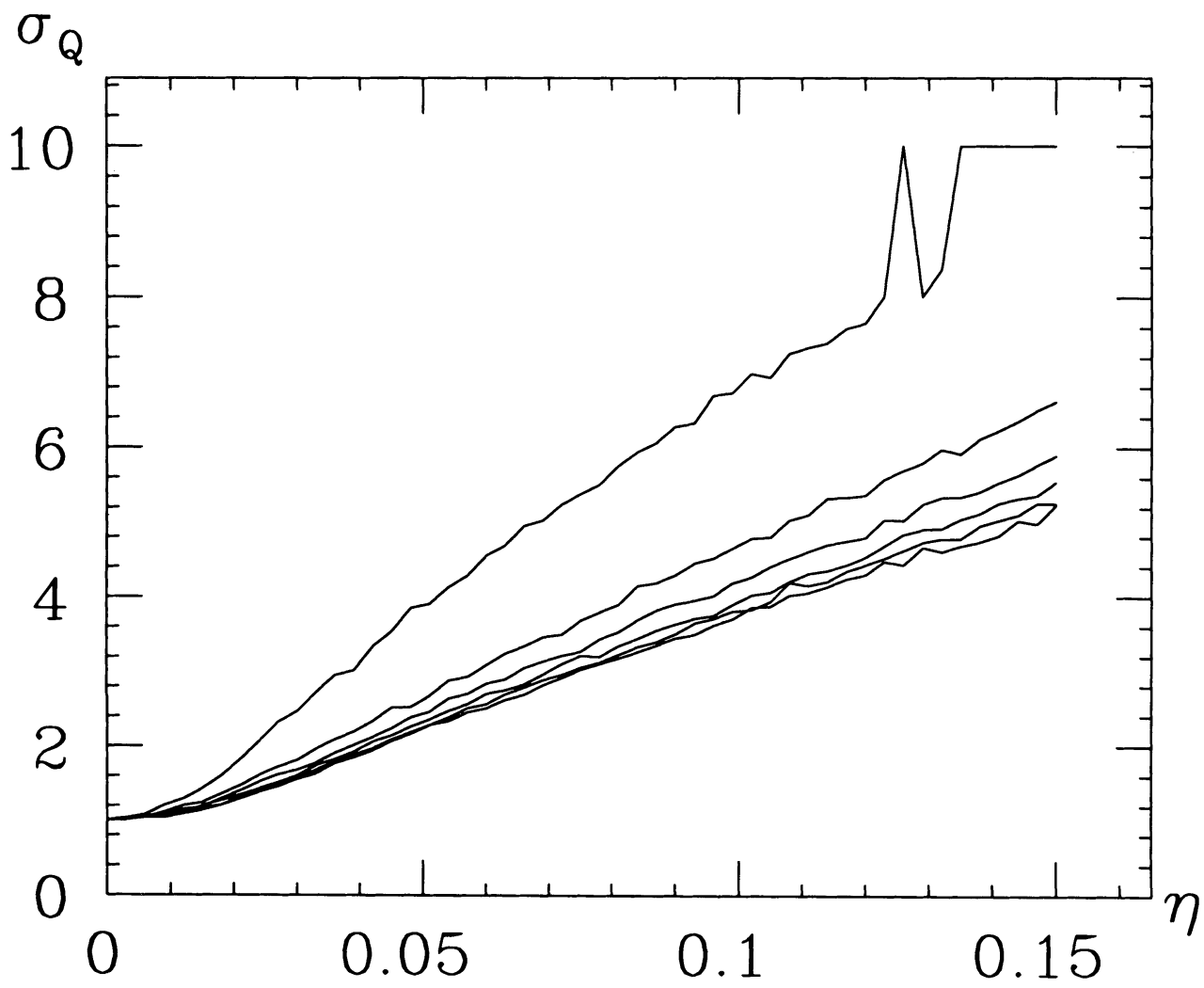

FIGURE 1: Transverse beam size $\sigma_{Q}$ as a function of $\eta$ with different numbers of slices, $L$ : those with $L=1$ to 6 lie from top to bottom. Parameters: $\nu_{x}=0.15, \nu_{s}=0.085, T_{\epsilon}=200$ and $T_{x}=400, R_{1}=10, R_{2}=0$.

The ratio $R_{1}$ represents a geometrical effect of the floating collision point: beams have different sizes at different CP's. The ratio $R_{2}$ represents the energy kick associated with the transverse kick. For usual parameters of $e^{+} e^{-}$colliding rings, $R_{2}$ is very small: this is at most $10^{-2}$, representing the fact that the longitudinal emittance is much larger than the transverse emittance.

\subsection{Results of Simulation}

5.2.1 Dependence on the number of slices In Fig. 1, the rms transverse beam sizes

$$
\sigma_{Q}=\left(\left\langle Q^{2}\right\rangle-\langle Q\rangle^{2}\right)^{1 / 2}
$$

are shown as a function of $\eta$ with changing $L$. Since $R_{1}=10$ is large, the effects of the floating collision points are a little exaggerated.

For small values of $L$, the results depend on it significantly, being consistent with Ref. ${ }^{7}$. We may conclude that $L=5$ is sufficient. 


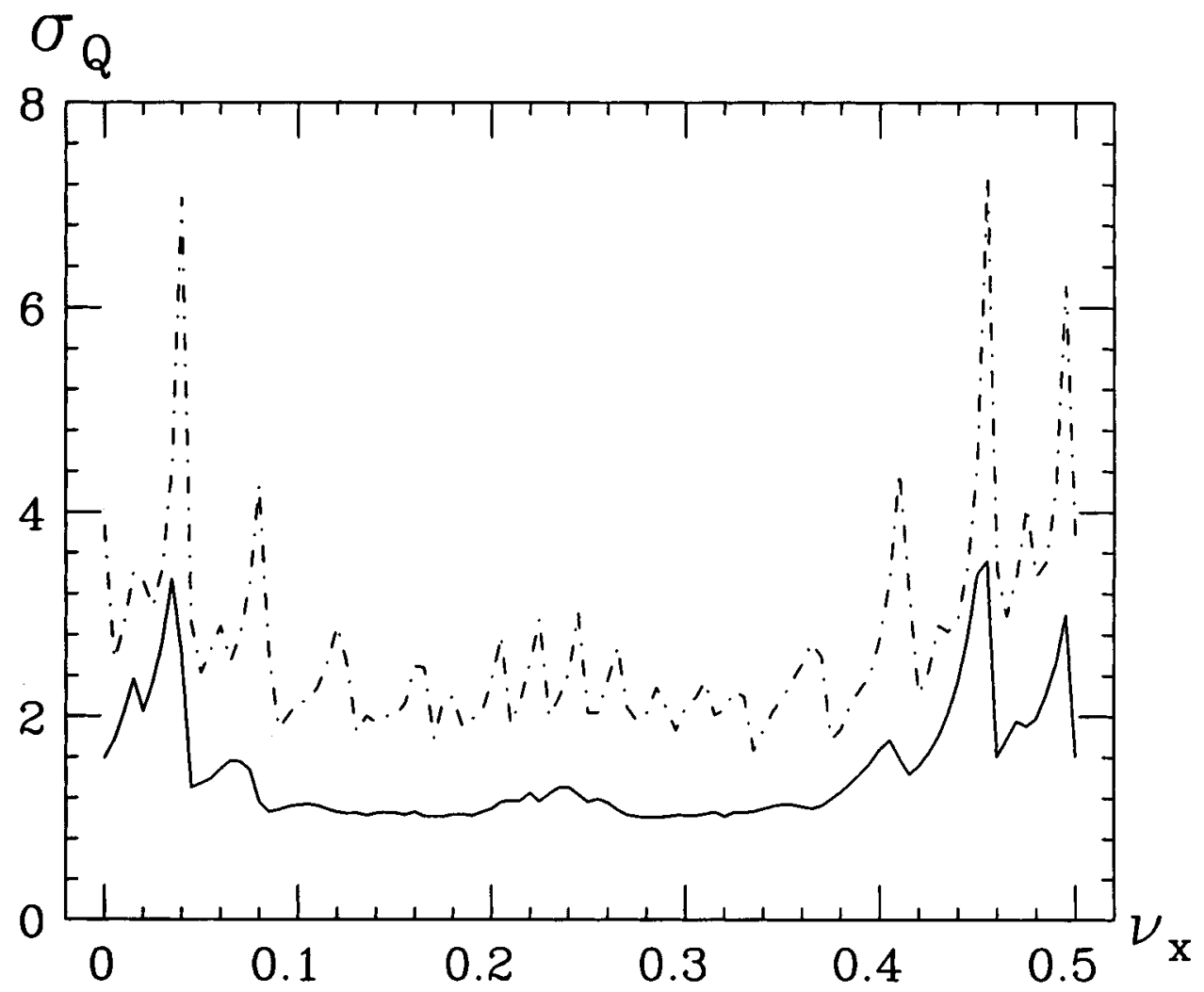

FIGURE 2: Beam size $\sigma_{Q}$ as a function of $\nu_{x}$ with different value of slices, $L: L=1$ (dotted line) and 5 (solid line). Parameters: $\nu_{s}=0.085, T_{\epsilon}=200$ and $T_{x}=400, \eta=0.05, R_{1}=1, R_{2}=0$.

5.2.2 Tune Dependence In Fig. 2, equilibrium transverse beam size is shown as a function of $\nu_{x}$. The synchro-betatron resonances near

$$
2 \nu_{x} \pm \nu_{s} \simeq \text { integers, }
$$

are the most dangerous. This comes from the bunch-length effects represented by $R_{1}$. The energy kick effect, represented by $R_{2}$ does not change the situation, except for the case of unrealistically large value of $R_{2}$. For comparison, we have shown the case with $L=1$ too. The reduction of the simulation results by the floating CP effect seems almost universal for various values of $\nu_{x}$.

5.2.3 Beam Blowup When the current, i.e. the $\eta$, increases, the beam sizes increase too. To parametrize this effect, let us define $\eta_{\infty}$ by the value of $\eta$ where $\sigma_{Q}=2$. The corresponding real beam-beam parameter, $\xi_{\infty}$, is four times as small as $\eta_{\infty}$, because we use the real beam size in the latter case. (Note that the $\eta$ is proportional to the current, whereas the $\xi$ is proportional to the luminosity, apart from the possible "hourglass effect"17,18. In Fig. 3, $\xi_{\infty}$ is shown as a function of $R_{1}$ for two very different values of the damping time. 


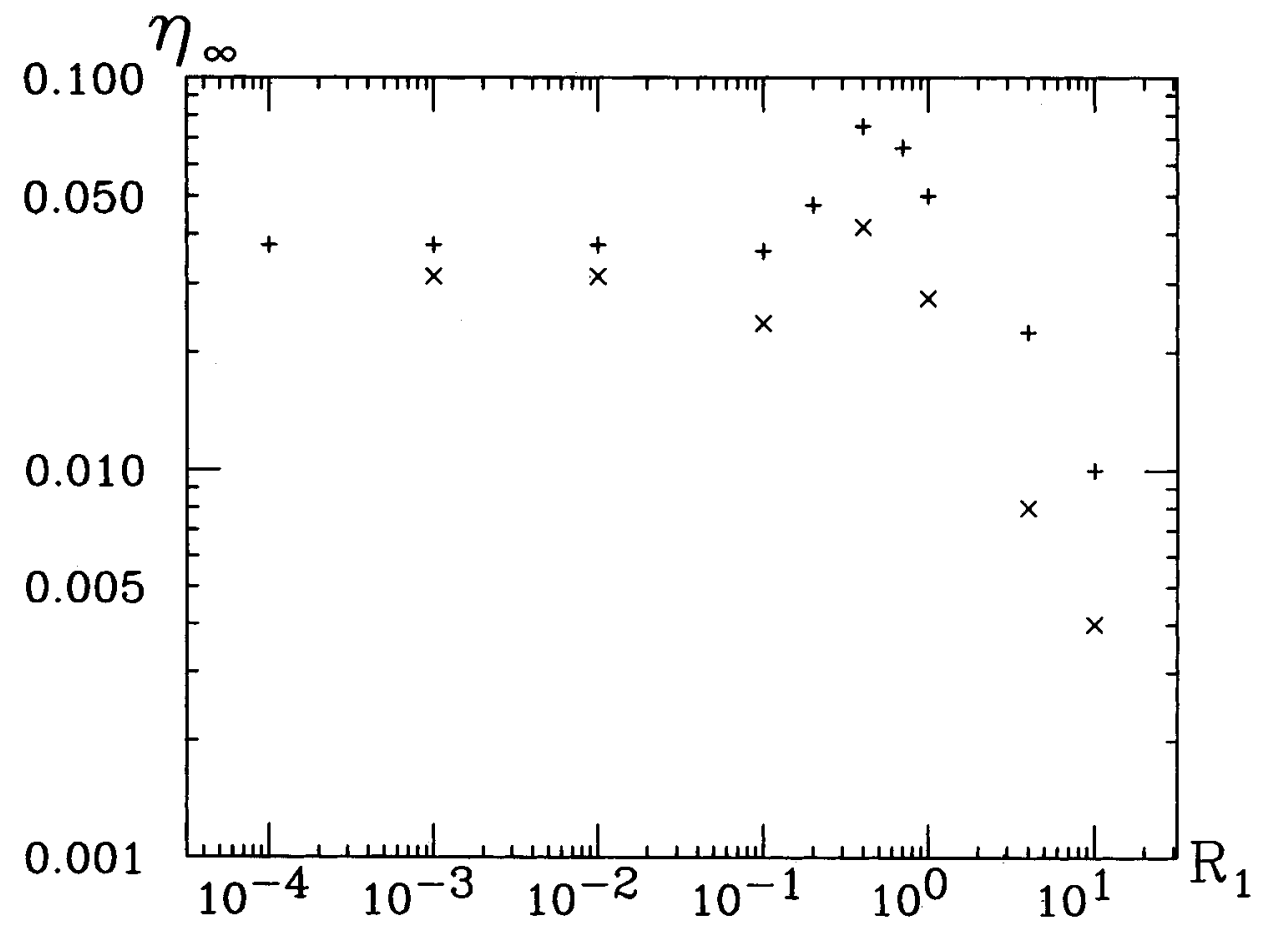

FIGURE 3: The maximum value of the nominal beam-beam parameter $\eta$ as a function of $R_{1}$. The parameters are $\nu_{x}=0.15, \nu_{s}=0.085,\left(T_{x}, T_{\epsilon}\right)=(4000,2000)(\times)$ and $(2000,200)(+)$ and $L=5$. The 'maximum' is defined such that $\sigma$ becomes twice as large as $\sigma_{0}$.

In this connection, it is interesting to note that, in Fig. 3,

$$
\xi_{\infty} R_{1} \simeq \text { const }
$$

holds for $R_{1} \lesssim 1$ and when $T_{x}$ and $T_{\epsilon}$ are fixed. This is consistent with a phenomenological observation ${ }^{19}$ that the limiting value of the disruption parameter, $D_{\infty}$, is almost universal. Here

$$
D_{\infty}=4 \pi \xi_{\infty} \frac{\sigma_{s}}{\beta_{x}^{0}}=8 \pi \xi_{\infty} R_{1} .
$$

Another interesting observation is that $\xi_{\infty}$ seems to have its maximum (perhaps a local maximum) at around $R_{1}=1 / 2$, which corresponds to $\beta_{x}=\sigma_{z}^{0}$. This fact seems to be consistent with the observation at $\mathrm{CESR}^{20}$.

5.2.4 Tail Distribution The multiparticle tracking results given thus far may appear to imply that $R_{2}$ is not an important factor and can be ignored at least for electron rings. Actually, however, the energy change may influence the long term behaviour of the particles. In particular, the tail distribution may be modified.

In Fig. 4, we show the transverse distribution $\rho(Q)+\rho(-Q)$ for different values of $R_{2}$. Even when $R_{2}=0$, the transverse distribution deviates from a Gaussian in the 


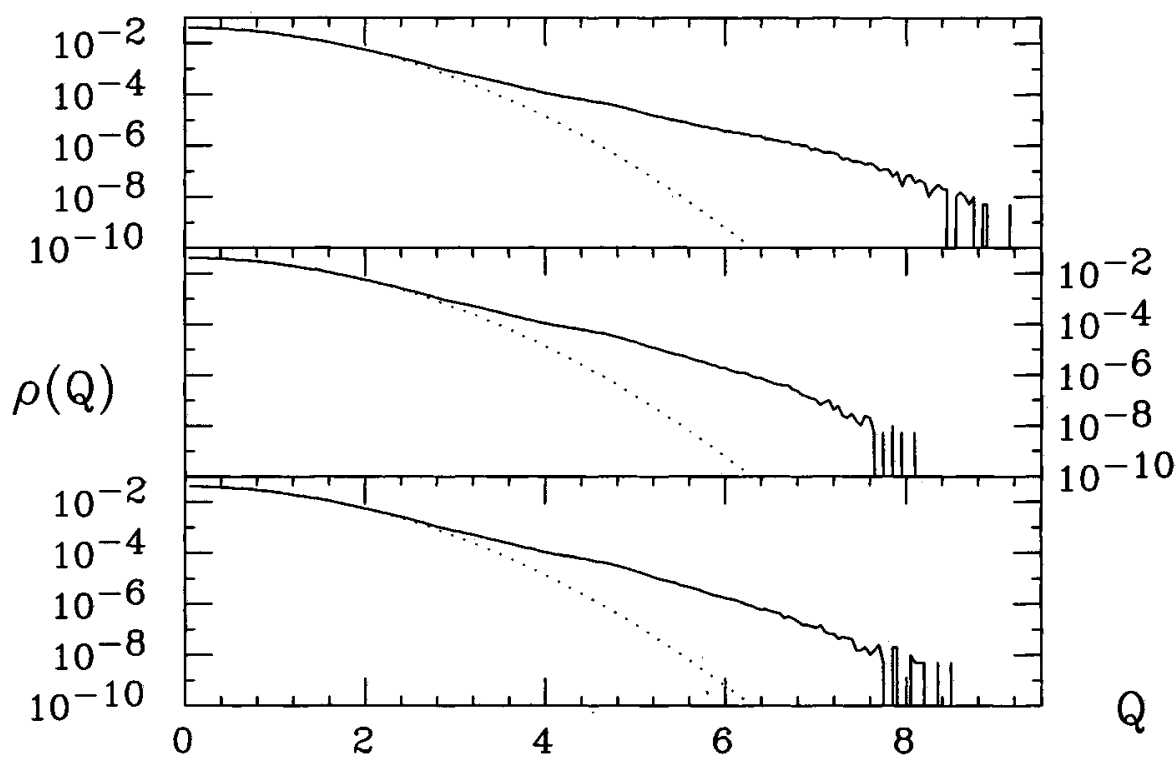

FIGURE 4: Transverse distribution for three values of $R_{2}: R_{2}=0$ (bottom), $R_{2}=0.01$ (middle) and $R_{2}=0.1$ (top). The horizontal axis is $Q$, the normalized distance from the origin, and the vertical the distribution in $Q$. The data was taken for 100 particles and through $2 \times 10^{6}$ turns. Parameters: $\nu_{x}=0.15, \nu_{s}=0.085$, $T_{\epsilon}=200$ and $T_{x}=400, \eta=0.1, R_{1}=0.5$ and $L=3$. Dotted lines correspond to the case $\eta=0$.

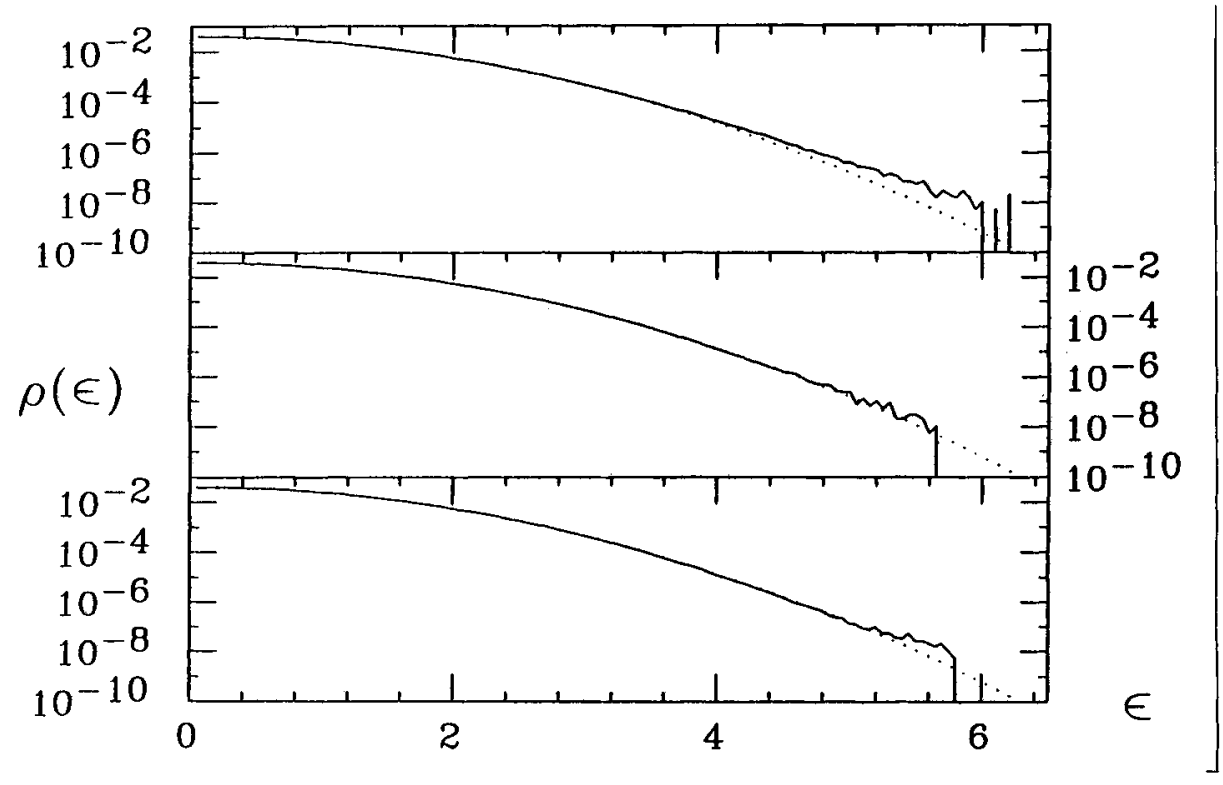

FIGURE 5: Energy distribution for three values of $R_{2}: R_{2}=0$ (bottom), $R_{2}=0.01$ (middle) and $R_{2}=0.1$ (top). The horizontal axis is $\mathcal{E}$ and the vertical the distribution in $\mathcal{E}$. The data was taken for 100 particles and through $2 \times 10^{6}$ turns. Parameters are the same as Fig. 4. Dotted lines correspond to the case $\eta=0$. 
tail. This is a well-known effect. We superposed the nominal $(\eta=0)$ distribution for comparison by dotted lines. The tail does not contribute to the rms beam sizes so that the nominal Gaussian fits the central region very well. The non-Gaussian tail in the transverse direction does not affect the luminosity, as long as the central part is not seriously modified. This, however, may influence the background to the detectors as well as the lifetime.

The effect of $R_{2}$ is remarkable in the further tail. It appears that the further tail for $R_{2}=0.01$ is smaller than that for $R_{2}=0$ : for $R_{2}=0.1$ it is larger.

We show similar graph for the energy distribution in Fig. 5. In this case, both $R_{1}$ and $R_{2}$ are important parameters. For $R_{2}=0$ (the bottom) the distribution should be exactly the nominal Gaussian: the tail part deviates from it only because of the finite number of data. Within this uncertainty, however, the $R_{2}$ has a certain effect on the tail. This tail is the direct consequence of the synchro-beam mapping.

\section{DISCUSSIONS}

\subsection{Chromatic Effects}

In interpreting the synchro-beam mapping, we have regarded

$$
p_{x} \sim \frac{\mathrm{dx}}{\mathrm{ds}} .
$$

Actually, there is a difference:

$$
\frac{\mathrm{dx}}{\mathrm{ds}}=\frac{p_{x}}{\sqrt{\left(\frac{|\boldsymbol{p}|}{\left|\boldsymbol{p}_{0}\right|}\right)^{2}-p_{x}^{2}-p_{y}^{2}}} \neq p_{x}
$$

The $\delta x^{\prime}$, should depend on the energy, $\epsilon$. This energy dependence of the transverse kick is one of the sources of chromaticity.

We have used the above approximation only in the virtual drift map: we should have used

$$
x(s)=x(0)+\frac{p_{x}}{\sqrt{\left(\epsilon+\frac{E_{0}}{c}\right)^{2}-p_{x}^{2}-p_{y}^{2}}} s .
$$

This will make a difference in $\delta x$ between particles having the same $p_{i}$ 's but different $\epsilon$ 's. This makes also a tiny change of $z$ (path length effect) during the whole beam-beam collision and thus brings a difficulty in using the concept of the longitudinal slice. What we have neglected is just this chromatic effect over the bunch length. This is, however, quite small when compared to the chromatic effect over the whole drift space. We can ignore it for the usual parameters of $e^{+} e^{-}$rings.

Our mapping, or the Hamiltonian, Eq. (23), does not depend on $\epsilon$. This should be so for the same reason that the Hamiltonian of a thin quadrupole magnet does not depend on $\epsilon$. We have assumed that there is no longitudinal magnetic field. With electric field 
and transverse magnetic field, the Lorentz equation says that the changes of $p_{x}, p_{y}$ and $|\boldsymbol{p}|$ do not depend on energy.

In the present scheme, thus, the chromatic aberration of the beam-beam force, if any, will appear only through the arc.

\subsection{Importance of the Synchro-Beam Mapping}

The synchro-beam mapping proposed in this paper is important for two reasons.

First, it provides us a $6 \times 6$ symplectic map. This enables us to install the beam-beam interaction in 6-dimensional tracking code. If the bunch-length effect is ignored, we can do it by using the $4 \times 4$ version. But if we want to include the bunch length effect, the only possible way is to use $6 \times 6$ formalism. The widely used technique of using $4 \times 5$ mapping, which includes the bunch-length effect on the transverse motion but does not include the energy-change depending on the transverse coordinates, explicitly breaks the symplecticity. Absolutely, this cannot be used for proton machines.

Relatedly, one may ask 'can we ignore $R_{2}$ in Eq. (89) for $e^{+} e^{-}$colliding rings?' The answer is 'yes', if we are interested only in the equilibrium rms beam sizes. The answer would be also 'yes', if we could stand the breaking of symplecticity. If we put $R_{2} \rightarrow 0$ in a coordinate frame used in the multiparticle tracking, Eq. (88), we cannot return to the original physical variable $x$ : the limit corresponds to a singular point of the transformation. Putting $R_{2}=0$ while keeping $R_{1} \neq 0$ corresponds to an unphysical situation that the energy spread is infinitely large but the bunch length is finite.

In presence of strong synchrotron radiation, one may say that the symplecticity is less important than for proton rings. For the rms beam sizes and the central distribution, it may be true, as shown by the simulation. But the statement still needs the proof. As we have shown in the simplified multiparticle tracking, in addition, for more delicate issues such as the tail distributions, however, the loss of $R_{2}$, i.e. the loss of the symplecticity may cause a serious errors even in the electron case.

\subsection{Conclusion}

The synchro-beam mapping is a fairly accurate, though not 'exact', model of the beambeam interaction. It is symplectic in 6-dimensional sense for an arbitrary distribution for the beams. It applies both to weak-strong and strong-strong cases. It has a reasonable physical interpretation. We of course do not reject less accurate treatments, such as $4 \times 4$ treatment or $4 \times 5$ treatment (longitudinal distribution can affect transverse dynamics, but not vice versa), as long as the synchro-beam effects seem ignorable. The claim on the luminosity is, however, increasing rapidly. Thus the beam-beam interaction should be studied more and more carefully. In this situation, we should use the most accurate method available to treat the beam-beam effects. The synchro-beam mapping is a good candidate for this purpose. More detailed and extended works should be relegated to the future, including the strong-strong simulation (it requires a tremendous CPU time to do this job reliablly enough) and long-term single particle tracking with lattice nonlinearity. 


\section{ACKNOWLEDGEMENTS}

This work started when K.H. and H.M. stayed at CERN. They thank members of the CERN SL-AP group for the hospitality extended to them. M. Bassetti is acknowledged for raising authors attention to the energy loss due to the electric field. They thank also M. A. Furman and A. Piwinski for sending them valuable comments.

\section{REFERENCES}

1. The most recent overview of these issues can be found, for example, in the Proc. Third Advanced ICFA Beam Dynamics Workshop on Beam-Beam Effects in Circular Colliders 29 May, 1989, I. Koop and G. Tumaikin, Editors, Institute of Nuclear Physics Publication (1990).

2. J. Augustin, Orsay, 36-69 (1969).

3. A. Piwinski, IEEE Trans Nucl.Sci. NS-24 1408 (1977).

4. Accelerator Design of the KEK B Factory, (Eds. S. Kurokawa, K. Satoh and E. Kikutani), KEK Report 90-24 (1991).

5. An Asymmetric B Factory - Conceptual Design Report, LBL PUB-5303, SLAC-372, CALT-68-1715, UCRL-ID-106426, UC-IIRPA-91-01(1991).

6. Feasibility Study for a B Meson Factory in the CERN ISR Tunnel, Ed. T. Nakada, CERN 90-02 (1990).

7. S. Krishnagopal and R. Siemann, Phys. Rev. D41,2312 (1990).

8. M. Bassetti, Longitudinal Energy Changes in a Bunch, LNF-T-105 (1978).

9. M. Bassetti, Come Migliorare gli Attuali Limiti di Luminosita', LNF-ARES-18 (1989).

10. K. Hirata , H. Moshammer, F. Ruggiero and M. Bassetti, CERN SL-AP/90-02 (1990).

11. K. Hirata , H. Moshammer, F. Ruggiero and M. Bassetti, Synchro-Beam Interaction, AIP Conference Proceedings No.214, 'Beam Dynamics Issues of High-Luminosity Asymmetric Collider Rings', (Ed. A.M.Sessler) p.389 (1990).

12. A. J. Dragt, in Physics of High Energy Accelerators, proceedings of the Summer School on High Energy Particle Accelerators, Fermilab, 1981, edited by R.A. Carrigan, I.R. Huson, and M. Month (AIP Conf. Proc. No. 87) (AIP, New York, 1982), p. 147.

13. M. Bassetti and G. Erskine, CERN ISR TH/80-06 (1980).

14. B. W. Montague, CERN ISR-GS 36 (1975).

15. B. Richter, Proc. Int. Symp. Electron and Positron Storage Rings, Saclay, 1966, page I-1-1.

16. K. Hirata and F. Ruggiero, Part. Accel. 28, 137 (1990).

17. G. E. Fischer, A Brief Note on the Effect of Bunch Length on the Luminosity of a Storage Ring with Low Beta at the Interaction Point, SPEAR-154 (1972).

18. SPEAR Storage Ring Group, Measurements on Beam-Beam Interaction at SPEAR, IEEE Trans. Nucl. Sci. NS-20, 3, 838 (1973).

19. S. Milton, Evidence of a Disruption Limit in Storage Rings, Paul Scherrer Institut Report PR-90-05, 1990.

20. D. H. Rice, AIP Conference Proceedings No.214, page 219 (1990). 